\title{
A Modular Integration of SAT/SMT Solvers to Coq through Proof Witnesses ${ }^{\star}$
}

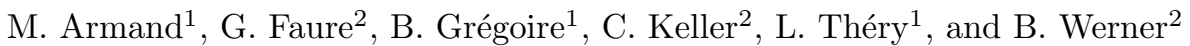 \\ 1 INRIA Sophia-Antipolis \\ \{Michael.Armand, Benjamin.Gregoire, Laurent. Thery\}@inria.fr \\ 2 INRIA - Saclay- ̂̂le-de-France at LIX, École Polytechnique \\ \{Germain.Faure, Chantal.Keller, Benjamin.Werner\}@inria.fr
}

\begin{abstract}
We present a way to enjoy the power of SAT and SMT provers in Coq without compromising soundness. This requires these provers to return not only a yes/no answer, but also a proof witness that can be independently rechecked. We present such a checker, written and fully certified in Coq. It is conceived in a modular way, in order to tame the proofs' complexity and to be extendable. It can currently check witnesses from the SAT solver ZChaff and from the SMT solver veriT. Experiments highlight the efficiency of this checker. On top of it, new reflexive Coq tactics have been built that can decide a subset of Coq's logic by calling external provers and carefully checking their answers.
\end{abstract}

\section{Introduction}

When integrating a technology like SAT/SMT solvers in type theoretical provers like Coq, one classically has the choice between two ways, which Barendregt and Barendsen [4] named the autarkic and the skeptical approach.

In the autarkic approach, all the computation is performed in the proof system. In this case, this means implementing a whole, sufficiently efficient, SAT/SMT solver as a Coq function, and then proving it correct. This approach is followed in the Ergo-Coq effort [10].

In the skeptical approach, the external tool, here the SAT/SMT solver, is instrumented in order to produce not only a yes/no answer but also a proof witness, or a trace of its computation. It is the approach we follow here. The main contribution of the paper is to propose a modular and effective checker for SAT and SMT proof witnesses, written in Coq and fully certified.

In general, the choice between the autarkic and the skeptical approach depends on the considered problem. Typically, when the problem is solved by a greedy algorithm or something similar requiring no backtracking, the autarkic approach is generally to be preferred. In the case of SAT/SMT solvers, where a lot of time is devoted to actually finding the proof path, the skeptical approach can have an edge in terms of efficiency. Another advantage of the skeptical approach may be that it requires much less effort to certify a checker only than

* This work was supported in part by the french ANR DECERT initiative. 
a whole prover. A difficulty is that the external prover and the internal checker have to speak a common language; indeed, finding the best possible format for the proof witnesses is the crucial step which determines the whole architecture of this prover-checker interaction.

Let us note that we see two benefits of this work. The first one being, as mentioned above, the addition to Coq of powerful and yet sound automation tactics. A second one is that it gives a means to enhance the reliability of automatic provers, by offering the possibility to have their results checked, a posteriori in Coq.

One keypoint for success in computational proofs is making the best possible usage of the, somewhat limited, computational power available inside the prover (Coq). This concern underlies the whole work presented here. An important advantage is the new addition to Coq of updatable arrays [3] which we use extensively in this work.

A wholly different approach to the integration of SAT/SMT to provers is to transform the proof witnesses into deductions. This approach has been taken for the Isabelle and HOL Light provers. We provide some comparison in Section 7.

The paper is organized as follows. The next section recalls the basic principles of SAT and SMT solvers. Section 3 describes the modular structure of the checker written in Coq; Sections 4 and 5 detail its different components, dealing respectively with the SAT parts, and two specific theories. Part 6 describes how the different parts are linked together in order to provide a practical tool. Finally, Section 7 is devoted to benchmarks and comparison with other works.

The source code of the checker and information on its usage can be found online [1].

\section{The SAT and SMT problems}

\subsection{SAT solvers}

SAT solvers deal with propositional formulas given in Conjunctive Normal Form $(\mathrm{CNF})$; they decide whether or not there exists an assignment of the variables satisfying the formula. We recall basic definitions. A literal is a variable or its negation, a clause is a disjunction of literals, noted $l_{1} \vee \cdots \vee l_{n}$. Finally, a formula in CNF is given by a finite set of clauses $\mathcal{S}$, seen as their conjunction.

A valuation $\rho$ associating a Boolean to each variable straightforwardly induces an interpretation of a set of clauses $\left(\llbracket S \rrbracket_{\rho}\right)$ as a Boolean. A set of clauses $\mathcal{S}$ is satisfiable if and only if there exists a valuation $\rho$ such that $\llbracket \mathcal{S} \rrbracket_{\rho}=\top$. Conversely, $\mathcal{S}$ is unsatisfiable if and only if for any valuation $\rho, \llbracket \mathcal{S} \rrbracket_{\rho}=\perp$.

Modern SAT solvers rely on variants of the DPLL algorithm which can be customized to generate a proof witness [12]. The witness is:

- either an assignment of the variables to $T$ and $\perp$ in order to satisfy all the clauses, in the case where the set of clauses is satisfiable;

- or a proof by resolution of the empty clause, in the case where the formula is unsatisfiable. 
We recall that the refutationally complete resolution rule is:

$$
\frac{v \vee C \quad \bar{v} \vee D}{C \vee D}
$$

where $v$ is called the resolution variable. A comb tree is a tree where at least one child of every inner node is a leaf. A comb tree of resolution rules is called a resolution chain.

From the point of view of result certification, the case of unsatisfiability is the more challenging one. The format used by most SAT solvers for proof witnesses of unsatisfiability is a list of resolution chains. This list should be understood as a shared representation of the resolution tree: each resolution chain derives a new clause (that can be used later by other resolution chains), and the last resolution chain should derive the empty clause. It corresponds exactly to a subset of the learned clauses that the algorithm encountered during its run.

\section{$2.2 \quad$ SMT solvers}

SMT solvers decide an extension of the SAT problem in which positive literals are not only Boolean variables but also atomic propositions of some first-order theory (possibly multisorted). Given a signature $\Sigma$ containing simple types, and function and predicate symbols with their types, a theory $\mathcal{T}$ is a set of formulas of type bool written using this signature, variables and logical connectives. Those formulas are called theory lemmas.

The standard architecture for SMT solvers is an interaction between a SAT solver and decision procedures for the theories [12]. The SAT solver generates models and the theory solvers try to refute them. When a SAT model is consistent with all the theories, the initial problem is found satisfiable. Otherwise, a new clause corresponding to a theory lemma is added to the SAT problem in order to rule out the model. The SAT solver can then be called again to generate another model. Since there are only a finite number of SAT models, this enumeration eventually terminates. If the empty clause is derived by the SAT solver, the initial problem is unsatisfiable.

In this setting, a proof witness for unsatisfiability is a resolution tree of the empty clause where leaves are not only initial clauses but also theory lemmas.

To our knowledge, at least three existing SMT solvers can deliver informative proof witnesses of this kind: Z3, CVC3 and veriT (some other solvers provide less informative witnesses). Although there are some output format differences, each of these three provers does return a resolution tree with theory lemmas in the case of unsatisfiability. They also give witnesses for satisfiability.

\section{A modular and efficient Coq checker for SAT and SMT proof witnesses}

We have developed a general framework to verify SAT and SMT proof witnesses for unsatisfiability proofs. During the SAT/SMT process, new clauses are generated until reaching the empty clause. These new clauses are either propositional 
consequences of the initial clauses, theory lemmas, or related to the CNF conversion and to various simplifications. A small certificate is generated to explain how each of the new clauses that are useful to obtain unsatisfiability was produced.

Our checker is defined by bringing together small checkers, each dedicated to one aspect of the verification of the resolution tree (the propositional reasoning, the theory lemmas of a given theory, the CNF conversion etc.). This modularity is a key aspect of our work. Small checkers are then independent pieces of code that can be composed in a very flexible way. Section 4.1 is dedicated to checking resolution chains, Section 4.2 to checking CNF computation. For theory lemmas, Section 5.2 describes what has been done for congruence closure and Section 5.3 for linear integer arithmetic. In each section, we present exactly the certificate format, how the checker works and is proved correct. The actual connection between Coq and the SAT and SMT provers is presented only later in Section 6 .

The common aim underlying these different parts is preserving efficiency, in time and space. The main difficulty is the very large number of clauses that may need to be handled. We therefore strongly rely on the new persistent arrays feature of Coq, described in [3]. Schematically, checkers can be understood as sharing a global state, holding the current set of clauses, and implemented by an array. One typical optimization will be to keep this array as small as possible, by re-using a cell as soon as the clause it holds is known to be not used anymore for further computations.

In order to achieve modularity, we restrict ourselves to a very lightweight interface for the small checkers. Our implementation is based on four main data types: $\mathcal{S}, \mathcal{C}$, clauseld, and scertif. The first one, $\mathcal{S}$, represents the state. Initially, the only clause in the state is the singleton clause that contains the formula to be proved unsatisfiable. The type for clauses is $\mathcal{C}$. An element of type clauseld is an identifier that refers to a clause. The get and set functions let us access and modify the state and the main checker only needs to be able to check whether a clause is empty, i.e it represents $\perp$ :

$$
\text { get }: \mathcal{S} \rightarrow \text { clauseld } \rightarrow \mathcal{C} \quad \text { set }: \mathcal{S} \rightarrow \text { clauseld } \rightarrow \mathcal{C} \rightarrow \mathcal{S} \quad \text { isFalse }: \mathcal{C} \rightarrow \text { bool }
$$

A small checker in our setting is just a Coq program that, given a state and a small certificate $c$, returns a new clause $C$. It is correct if the new clause that is produced is a consequence of the state $S$ :

$$
\text { for any interpretation } \rho, \llbracket S \rrbracket_{\rho} \Rightarrow \llbracket C \rrbracket_{\rho}
$$

The type scertif is an enumeration type that collects all the possible small certificates. Associated to this type, there is a dispatching function scheck that, depending on the small certificate it receives, calls the appropriate checker.

Since small checkers just generate clauses, the only information we have to provide when gluing together small certificates is where the new clauses have to be stored. Finally, at the end, the initial formula should be proved unsatisfiable, so the empty clause must have been derived. So, its actual location must be given by the main certificate. The type of such certificate is then

$$
\text { certif }:=\text { list }(\text { clauseld } * \text { scertif }) * \text { clauseld }
$$


The main checker is now trivially defined by

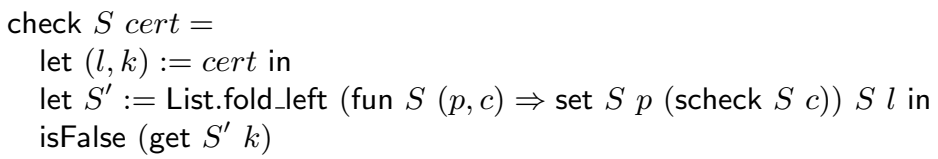

It takes an initial state $S$ and a certificate cert and sequentially calls small checkers to compute new clauses extending the state with the generated clauses. Provided all small checkers are correct and the check returns true and since satisfiability is preserved, reaching an absurd state implies that the initial state was indeed unsatisfiable.

As hinted above, the get and set functions are built upon the persistent arrays of Coq and one such array is used in the state to store clauses. clauselds are thus array indexes, i.e. 31-bit integers. So, access and update to the set of clauses are performed in constant time. Since we use arrays, in the following, (get $S n$ ) is written as $S .[n]$.

It is very unlikely that a given SMT solver will output exactly our kind of certificates. A pre-processing phase is required in order to translate proofwitnesses generated by the SMT solver into our format. In particular, the precise clause allocation that is required by our format is usually not provided by the SMT solver. Finding out such an allocation is a post-processing seen from the SMT solver, and a pre-processing seen from Coq. It involves techniques similar to register allocation in compilation. First, the maximal number of clauses that need to be alive at the same time is computed. Then, a cell is explicitly allocated to each clause, in such a way that two clauses that need to be alive at the same time do not share the same cell. In practice, this has a big impact on the memory required to check a certificate.

\section{Small checkers for SAT}

\subsection{A small checker for resolution chains}

As explained in Section 2.1, the SAT contribution is represented in the proof witness by chains of resolutions. The constructor (res_certif $\left[\left|n_{1} ; \ldots ; n_{i}\right|\right]$ ) in scertif represents these chains. Let $R\left(C_{1}, C_{2}\right)$ be the resolution between the clause $C_{1}$ and the clause $C_{2}$. Given this certificate and a state $S$, the corresponding small checker iteratively applies resolution to eventually produce the new clause $R\left(\ldots\left(R\left(S .\left[n_{1}\right], S .\left[n_{2}\right]\right), \ldots\right), S .\left[n_{i}\right]\right)$.

This efficient treatment of resolution chains requires a careful encoding of clauses and literals. First, we encode propositional variables as 31-bit integers. We follow the usual convention reserving location 0 for the constant true, which means that the interpretation of propositional variables $\rho$ always comes with the side-condition that $\rho(0)=$ true. Literals are also encoded as 31-bit integers, taking advantage of parity. The interpretation for literals is built such that:

$$
\llbracket l \rrbracket_{\rho}=\text { if even } l \text { then } \rho(l / 2) \text { else } \neg \rho(l / 2) .
$$


The point being that parity check and division by two are very fast since they are directly performed by machine integer operations as explained in [3].

Clauses are represented by lists of literals. The interpretation $\llbracket c \rrbracket_{\rho}$ of a clause $c$ is then the disjunction of the interpretation of its literals. The interpretation $\llbracket S \rrbracket_{\rho}$ of a state $S$ is defined as the conjunction of the interpretation of its clauses.

To give a concrete example, consider the interpretation of proposition variables: $\rho(0)=$ true, $\rho(1)=x, \rho(2)=y$. If $S=[|[2 ; 4] ;[5] ;[3 ; 4]|]$ we have

$$
\begin{aligned}
\llbracket S \rrbracket_{\rho} & =\llbracket[2 ; 4] \rrbracket_{\rho} \wedge \llbracket[5] \rrbracket_{\rho} \wedge \llbracket[3 ; 4] \rrbracket_{\rho}=\left(\llbracket 2 \rrbracket_{\rho} \vee \llbracket 4 \rrbracket_{\rho}\right) \wedge \llbracket 5 \rrbracket_{\rho} \wedge\left(\llbracket 3 \rrbracket_{\rho} \vee \llbracket 4 \rrbracket_{\rho}\right) \\
& =(\rho(1) \vee \rho(2)) \wedge \neg \rho(2) \wedge(\neg \rho(1) \vee \rho(2))=(x \vee y) \wedge \neg y \wedge(\neg x \vee y)
\end{aligned}
$$

In this setting, the interpretation of a set of clauses is always a CNF formula. A proof of unsatisfiability of this formula is the following chain of resolutions

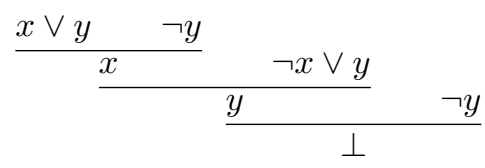

This corresponds in our format to certificate ([0, res_certif $[0 ; 1 ; 2 ; 1]], 0)$.

\subsection{Small checkers for CNF computation}

With our previous small checker, proof witnesses for SAT problems in CNF can be checked in Coq. The next step is to be able to verify the transformation of a formula into an equisatisfiable formula in CNF. This is usually done using a technique proposed by Tseitin [14]. This involves generating a new variable for every subterm of the formula; with these new variables, the CNF transformation is linear. It is this idea that we are going to implement in our setting. Naming subterms corresponds to a form of hash-consing. A hashed formula is either an atom, true, false, or a logical connective. Sub-formulas of connectives are literals (i.e., a variable or its negation):

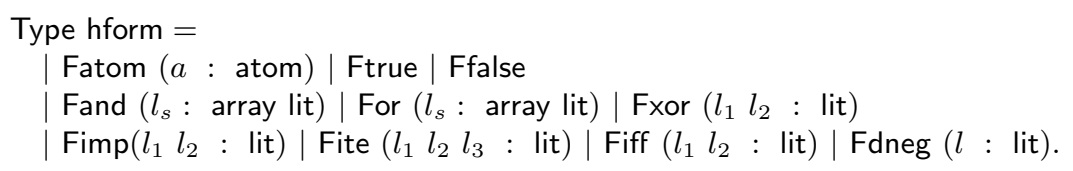

Note that the connectives Fand and For are n-ary operators which allows a more efficient subsequent computation. Note also that we have no primitive constructor for negation, which has to be pushed to the literals (with little cost, using the odd/even coding described above). However, double negation is explicit and primitive, in order to represent the formula $\neg \neg x$ faithfully.

For computation, the state of the checker is extended with a new array ftable containing the table of the hashed formulas. For example, the formula $\neg((x \wedge$ $y) \vee \neg(x \wedge y))$ can be encoded by the literal 9 using the formula table:

$$
\text { [| Ftrue; Fatom 0; Fatom 1; Fand [|2;4|]; For [|6;7|] |] }
$$

with the interpretation for atoms defined by $\rho_{A}(0)=x, \rho_{A}(1)=y$. Three things are worth noticing. First, the sub-formula $x \wedge y$ appears twice in the formula but 
is shared in the table (at location 3); indeed, this representation allows maximal sharing. Second, we have to ensure that our table does not contain infinite terms, so our state can be interpreted. This is done by preserving some well-formedness on the table: if a literal $m$ appears in the formula stored at location $n$, we always have $m / 2<n$. It is this condition that actually allows us to define the Boolean interpretation $\llbracket f \rrbracket_{\rho_{A}}$ recursively over the formula $f$, where $\rho$ is the interpretation of the variables.

Finally, the tables always have Ftrue at location 0 . The interpretation of propositional variables of the previous section is simply defined as $\rho(n)=\llbracket$ ftable. $[n] \rrbracket \rho_{\rho_{A}}$.

Tseitin identifies 40 generic tautology schemes used in the transformation to CNF. In the case of our example $\neg((x \wedge y) \vee \neg(x \wedge y))$, the transformation invokes the following tautology $\neg\left(A_{0} \vee \cdots \vee A_{i} \vee \cdots \vee A_{n}\right) \Rightarrow \bar{A}_{i}$. For each of these tautologies, we have written a specific test function which verifies that the corresponding tautology can actually be applied. In this case, the certificate is written (nor_certif $m i$ ) and the corresponding checker verifies that $S .[m]$ is a singleton clause $[k]$ with $k$ being odd and that ftable. $[k / 2]$ is a disjunction (For $\left.\left[\left|l_{1} ; \ldots ; l_{n}\right|\right]\right)$. If these conditions are met, it produces the clause $\left[\bar{l}_{i}\right]$ if $i<n$. If the verification fails, the true clause is returned. This trick of using the true clause as default will be used for all the other small checkers.

The full certificate of unsatisfiability for our example is then:

$$
([(1, \text { nor_certif } 00) ;(0, \text { nor_certif } 01) ;(0 \text {, res_certif }[|1 ; 0|]], 0) \text {. }
$$

The computation of the final set of clauses proceeds like this:

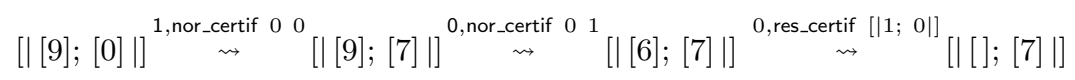

At the end, we find the empty clause at location 0 , which ensures the initial formula is unsatisfiable.

Let us finally remark that our format is compatible with lazy CNF transformation and also that it is possible to delegate the CNF computation to the SMT solver.

\section{Small checkers for congruence closure and linear arithmetic}

\subsection{Refining the term representation}

In order to handle theories, we need to provide a proper representation for atoms. Atoms can represent objects of different types, so we also need a proper representation for types. Theories like EUF manipulate uninterpreted functions, so we also need uninterpreted base types. Here is our representation:

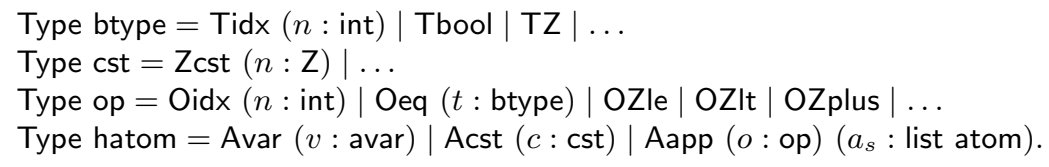


As for formulas, our encoding uses a table atable, so the type atom is an abbreviation for int. A hashed atom is either a variable (Avar), a constant of a theory (Acst), or an application of an operator to its arguments (list atom). Operators are uninterpreted functions or predicates (Oidx), or a function (OZplus) or a predicate (OZle) of a given theory. Base types are either uninterpreted (Tidx) or a type of a given theory (TZ).

To illustrate our representation, let us consider the formula $f x<1 \vee g(y+$ $1)<1$ over the Coq integer $Z$ where the Coq type of $x$ is $\alpha$ and is left uninterpreted. We have the following tables:

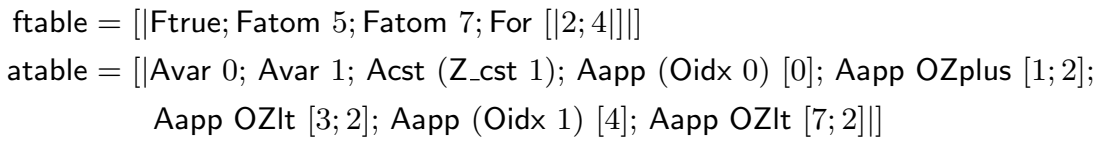

Interpreting types is easy. We just need a table ttable associating a Coq type to every type index. We denote by $\llbracket T \rrbracket_{t}$ the interpretation of a base type $T$ with respect to this table. Interpreting atoms is more difficult, since we must build well-typed Coq terms. In particular, different elements of the table may be interpreted into different types. Therefore, our interpretation function returns a dependent pair $(T, v)$ where $T$ has type btype and $v$ has type $\llbracket T \rrbracket_{t}$. The interpretation of atoms $\llbracket A \rrbracket$ uses two tables. The first one (vtable) is a valuation associating $(T, v)$ to a variable index. The second one (otable) associates a pair $\left(\left(\left[T_{1}, \ldots, T_{n}\right], T\right), f\right)$ to an operator index, where $T_{i}, T$ have type btype and $f$ has type $\llbracket T_{1} \rrbracket_{t} \rightarrow \ldots \llbracket T_{n} \rrbracket_{t} \rightarrow \llbracket T \rrbracket_{t}$. With these tables, defining the interpretation $\llbracket A \rrbracket$ is straightforward. We simply check that all applications are well-typed, if not we return (Tbool, true). This makes our interpretation a total function. Here are the three tables used by the interpretation for the previous example:

$$
\begin{aligned}
\text { ttable } & =[|\alpha|] \\
\text { vtable } & =[|(\operatorname{Tidx} 0, x) ;(\mathrm{TZ}, y)|] \\
\text { otable } & =[|(([\mathrm{Tidx} 0], \mathrm{TZ}), f) ;(([\mathrm{TZ}], \mathrm{TZ}), g)|]
\end{aligned}
$$

The interpretation of atoms of the previous section is simply defined as $\rho_{A}(a)=$ 【atable. $[a] \rrbracket$.

We need some side conditions on the different tables to be able to complete the proof of our small checkers. First, the hashed atom contained at position $k$ of the atable should refer to atoms strictly smaller than $k$ (this ensures that the interpretation terminates). Second, the atable should only contain well-typed hashed atoms with respect to vtable and otable. This last condition allows to reject formulas like $\neg(1=$ true $) \vee \neg($ true $=2) \vee(1=2)$ which is correct from the transitivity point of view but is interpreted in Coq by false $\vee$ false $\vee 1=2$

\subsection{A small checker to compute congruence closure}

The theory of congruence closure is at the heart of all SMT solvers. In our term representation, equality is represented as a binary operator (Oeq) that is parameterised by the representation of the type on which equality operates. 
Consider the proof of unsatisfiability of the formula $\neg(f a=f b) \wedge b=c \wedge a=c$, which belongs to the congruence closure fragment. It creates the following tables:

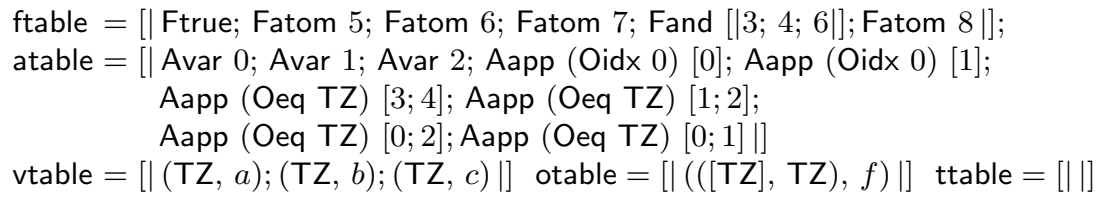

where the formula is at location 4 of ftable. Note that location 5 is not necessary to encode the formula but for its proof (the same thing happens for the location 8 of atable). This is explained later.

Our checker is only capable of producing clauses obtained by instantiating one of these three theorems:

- transitivity: $x_{1} \neq x_{2} \vee \cdots \vee x_{n-1} \neq x_{n} \vee x_{1}=x_{n}$

- function congruence: $x_{1} \neq y_{1} \vee \cdots \vee x_{n} \neq y_{n} \vee f x_{1} \ldots x_{n}=f y_{1} \ldots y_{n}$

- predicate congruence: $x_{1} \neq y_{1} \vee \cdots \vee x_{n} \neq y_{n} \vee \neg P x_{1} \ldots x_{n} \vee P y_{1} \ldots y_{n}$

The small certificates are (eq_trans $c$ ), (eq_congr $c$ ) and (eq_congr_pred $c$ ) where $c$ represents the candidate clause to be produced. This explains why the tables of our previous example had more than the atoms of the initial formula. They also contain the atoms of the theory lemmas. The small checkers for these certificates only have to verify that $c$ is indeed an instantiation of their corresponding theorem. For instance, the small checker for eq_trans $\left[l_{1} ; \ldots ; l_{n} ; l\right]$ verifies that:

$-l$ is even and each $l_{i}$ is odd;

- $l / 2$ refers to Aapp (Oeq $t)[a ; b]$ and each $l_{i} / 2$ refers to Aapp (Oeq $\left.t_{i}\right)\left[a_{i} ; b_{i}\right]$;

- $a$ equals $a_{1}, b$ equals $b_{n}$ and for $1 \leq i<n, a_{i}$ equals $b_{i+1}$.

Note that all the equality tests over atoms are just equalities over integers thanks to our maximal sharing of atoms. Furthermore, we do not need to check type equality between the $t_{i}$ since the small checkers assume that the atom table is always well-typed.

Our modular checker can combine these three simple rules and the resolution checker to derive the empty clause from an unsatisfiable formula. In our example, the checker starts with the initial formula $\neg(f a=f b) \wedge b=c \wedge a=c$. After evaluating the part of the certificate dedicated to CNF computation, the state contains the clauses $[|[3] ;[4] ;[6] ;[0] ;[0]|]$ and what is left to be evaluated is

$$
([(3 \text {, eq_trans }[5 ; 7 ; 10]) ;(4 \text {, eq_congr }[11 ; 2]) ;(0 \text {, res_certif }[|3 ; 1 ; 2 ; 4 ; 0|])], 0) \text {. }
$$

The computation then proceeds like this:

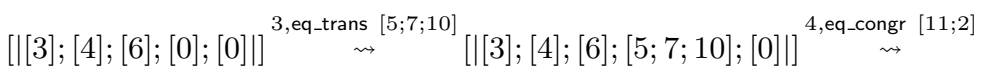

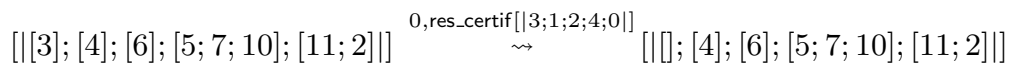




\subsection{A small checker for linear arithmetic}

The tactic lia [5] of Coq proves any valid formula of linear arithmetic. It already contains a checker based on Farkas' certificates:

$$
\text { lia_check : lia_formula } \rightarrow \text { lia_certif } \rightarrow \text { bool, }
$$

Note that lia uses a different representation (lia_formula). It also provides a proof of correction for this checker. We choose to use lia_check to build a small checker for our modular interface. Thus, the small certificate for linear arithmetic is simply (lia_certif $c F$ ) where $c$ is the candidate clause and $F$ of type lia_certif.

In order to validate the clause $c$, the small checker first calls the function lia_formula_of to translate $c$ into an equisatisfiable formula $f$ of type lia_formula, and then calls (lia_check $f F$ ). The correctness of this small checker relies on the correctness of the lia checker and on the correctness of our translation.

\subsection{The simplifier small checker}

For more efficiency, most SMT solvers use on-the-fly term rewriting and simplification and do not give proof witnesses for these simplifications. Furthermore, sometimes the formula needs to be preprocessed before being sent to an external solver, again for efficiency reasons. In consequence, the formula $f^{\prime}$ proved by the proof witness can be slightly different from the initial formula $f$ one wanted to prove. We have thus developed a dedicated small checker that verifies that a formula $f$ is equivalent to $f^{\prime}$. Our checker is able to prove equivalence through associativity of conjunction and disjunction, double negation, symmetry of equality, and simple rewriting of linear inequations (such as: $a \geq b \equiv b \leq a$ ). It is implemented by a simple simultaneous recursive descent of $f$ and $f^{\prime}$. Only the symmetry of equality requires some backtracking.

\section{Building a Coq tactic}

To build an actual tactic out of our certified checker, we follow the usual steps for reflexive tactics. The first step is reification: given a formula $f$ in Coq on a decidable domain, we have to build 5 tables and a literal $l$, such that the interpretation of $l$ with respect to these tables is $\neg f$. The second step is to find a certificate that shows that $[l]$ is unsatisfiable. This is done by calling the SAT or SMT solver. We need to translate the problem into the solver input format. Then, the solver returns a proof witness, that we transform into a certificate.

During the first translation, we sometimes need to do some pre-processing. For example, ZChaff only accepts CNF formulas, so the CNF transformation is done before sending it. Also, the CNF transformation of veriT is more efficient if disjunctions and conjunctions are considered as n-ary operators (and not binary like in (oq), so we flatten the formula before sending it. The justification of this pre-processing is the prelude of the certificate.

The transformation of proof witnesses into certificate requires more work. We first need to update our tables so they contain all the formulas of the theory 
lemmas. Second, we have to transform each step of the proof witness into a sequence of small certificates. In the easiest cases, the solver gives exactly what we expect. This is the case, for instance, of the resolution chains produced by SAT solvers and the CNF transformation produced by veriT. In other cases, very lightweight modifications are necessary. For instance, the format for congruence closure of veriT automatically removes duplicates literals. It generates $\neg x=$ $y \vee f x x=f y y$ while our certificate expects $\neg x=y \vee \neg x=y \vee f x x=f y$ y. Finally, in the worst cases, we may have to rebuild completely the certificate. This is the case for veriT where theory lemmas for linear arithmetic come without justification. In this precise example, we use the external solver of lia to produce the Farkas' certificate.

Finally, the compactness of the certificate is very important. So, when it is not done by the solver, we prune it by removing all the justifications of unused clauses. It is during this phase that we compute the minimal required size of the array of clauses and perform clause allocation.

This work results into two new Coq tactics called zchaff and verit .

\section{Results and comparison with other works}

\subsection{Related works}

The work presented here extends what is described in [3] in several ways. First, we complemented the SAT checker with checkers for CNF computation, congruence closure, differential logic and linear arithmetic. To do so with a great modularity, the format of certificates has been rethought: the idea of small and main checkers makes it easier to add new theories. Second, we can formally check proof witnesses generated by the veriT solver, which combines all these theories. Finally, we use our checker to enhance the automation of Coq by defining new safe reflexive decision procedures.

Several SAT and SMT solvers have been integrated in LCF style interactive theorem provers including CVC Lite in HOL Light [11], haRVey in Isabelle/HOL [8], Z3 in $\mathrm{HOL}$ and Isabelle/HOL [6]. To our knowledge, our work is the first integration relying on proof witnesses in a proof assistant based on Type Theory. In the following, we will focus on the comparison with proof reconstruction in Isabelle/HOL of ZChaff [15] and Z3 [6] (this corresponds to the state-of-the-art). We point out that comparison for theories has to be considered with care since we do not use the same SMT solver.

Another approach is to write the SAT or SMT solver directly inside the proof assistant and formally prove its correctness. This is the approach followed in [10]. It has the advantage to validate the algorithms at work in the prover but is sensitive to any change, any optimization in the proof search. We compare the two approaches.

\subsection{Experiments}

All the experiments have been conducted on an Intel Quad Core processor with 2.66GHz and $4 \mathrm{~Gb}$ RAM, running Linux. Our code which served for the experi- 
ments is available online [1]. It requires the native version of Coq [7]. It represents around 6,000 lines of Coq code and 8,000 lines of Ocaml code. The Coq code for our shared term representation is about 1,000 lines, the SAT part 1,200, the CNF part 1,500, the EUF 600, the LIA part 1,500 and the simplifier part 500. The complete checker corresponds to 1,000 lines of Coq code, the other 5,000 are for specifications and proofs.

SAT verification We first compare our combination of the main checker with the small checker of resolution chains for ZChaff in Coq with proof reconstruction for ZChaff in Isabelle/HOL written by Alwen Tiu and Tjark Weber. We use Isabelle 2009-1 (running with Poly/ML 5.2) and ZChaff 2007.3.12.

We run ZChaff on a database of 151 unsatisfiable industrial benchmarks from SAT Race'06 and '08 with a timeout of 300 seconds. These benchmarks range from 300 to 2.3 million variables and from 1,800 to 8.9 million clauses. When ZChaff succeeds in the given time, it produces a proof witness whose size range from $41 \mathrm{~Kb}$ to $205 \mathrm{Mb}$. In that case, we run our checker and the Isabelle/HOL checker on it with a timeout of 300 seconds. Table 1 presents the number of benchmarks solved by ZChaff, and among them, the number of proof witnesses successfully checked by Isabelle/HOL and Coq. The times are the mean of the times for the 57 benchmarks on which ZChaff, Coq and Isabelle/HOL all succeeded, in seconds. Errors in Isabelle/HOL were due to timeouts.

It appears that Coq can check all the proof witnesses given by ZChaff in the given time. This is not surprising since our checker appears to be faster than ZChaff itself. However, the Isabelle/HOL checker is slower than ZChaff, which explains that only $72 \%$ of the proof witnesses can be checked without timeout.

The three curves on the left of Figure 1 present the number of benchmarks solved along the time by ZChaff, Isabelle and Coq. It clearly shows that the Coq checker is far faster verifying results than ZChaff is building them; the main time consumed by our combination is taken by ZChaff. However, the limiting factor of the ZChaff and Isabelle/HOL combination is Isabelle/HOL.

SMT verification We now compare our combination of the main checker with the small checkers of resolution chains, CNF computation, congruence closure, differential logic and linear integer arithmetic for veriT in Coq with proof reconstruction for Z3 in Isabelle/HOL written by Sascha Böhme and Tjark Weber. We use Isabelle 2009-1 (running with Poly/ML 5.2), Z3 2.19 and the development version of veriT.

We took a database of unsatisfiable industrial benchmark from the SMTLIB [2] for theories QF_UF (congruence closure), QF_IDL (differential logic) and QF_LIA (linear integer arithmetic). It is important to notice that veriT is not completely proof producing for QF_LIA, so we selected a subset of the benchmarks where veriT returns either unknown or unsatisfiable with a proof witness. On the one hand, we run veriT, followed by our Coq checker when veriT succeeds. On the other hand, we run Z3, followed by the Isabelle/HOL checker when Z3 succeeds. Each run has a timeout of 300 seconds. The mean of the 


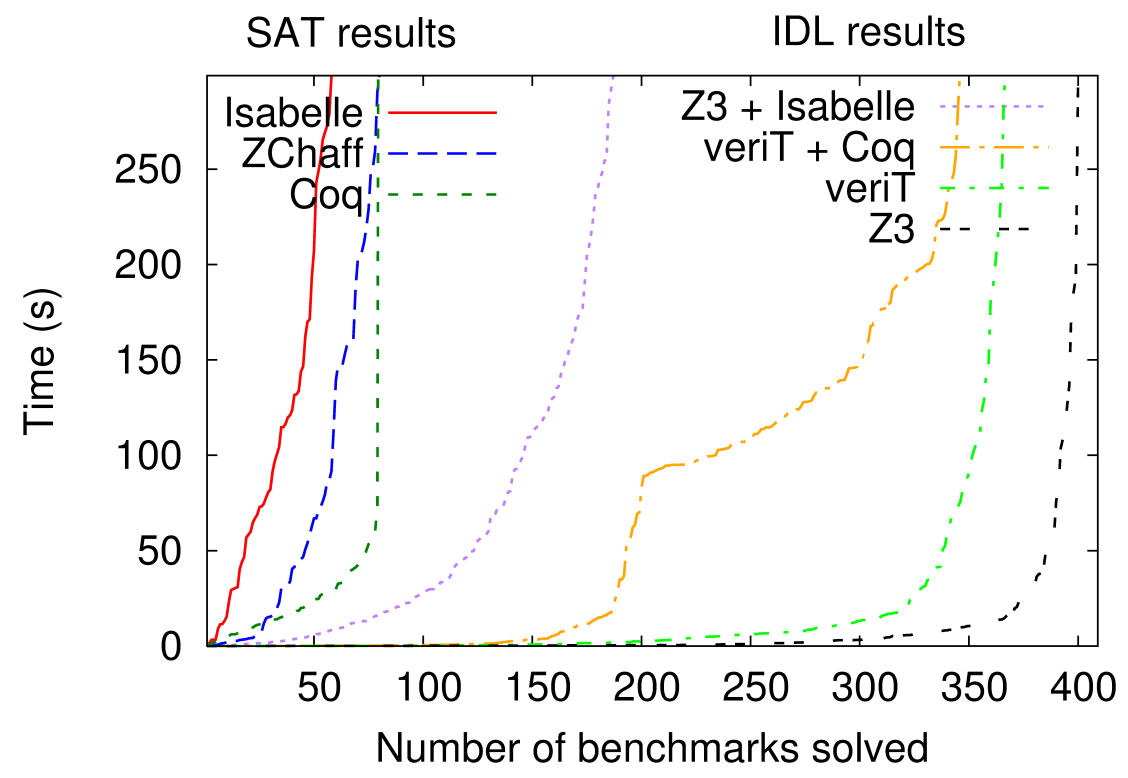

Figure 1: Experiments on industrial benchmarks

\begin{tabular}{|c|c|c|c|c|c|}
\hline \multicolumn{2}{|c|}{ Solved ZChaff } & \multicolumn{2}{|c|}{ Isabelle/HOL checker } & \multicolumn{2}{|c|}{ Coq checker } \\
\hline \# $\%$ & Time & \#\% & Time & $\# \%$ & Time \\
\hline \begin{tabular}{l|l|}
79 & 52
\end{tabular} & 51.9 & \begin{tabular}{|l|l|}
57 & 38 \\
\end{tabular} & 100. & 7952 & 17 \\
\hline
\end{tabular}

Table 1: SAT benchmarks

\begin{tabular}{|c|c||c|c|c||c|c|c||c|c|c||c|c|c|}
\hline \multicolumn{2}{|c||}{ Benchmarks } & \multicolumn{3}{c||}{ Solved Z3 } & \multicolumn{3}{c||}{ Solved veriT } & \multicolumn{3}{c||}{ Isabelle/HOL checker } & \multicolumn{3}{c|}{ Coq checker } \\
\hline Logic & $\#$ & $\#$ & $\%$ & Time & $\#$ & $\%$ & Time & $\#$ & $\%$ & Time & $\#$ & $\%$ & Time \\
\hline QF_UF & 1852 & 1834 & 99 & 2.5 & 1816 & 98 & 6.5 & 1775 & 96 & 25.8 & 1804 & 97 & 1.4 \\
\hline QF_IDL & 409 & 402 & 98 & 0.6 & 368 & 90 & 6.3 & 190 & 46 & 55.2 & 349 & 85 & 37.8 \\
\hline QF_LIA & 116 & 107 & 92 & 0.7 & 98 & 84 & 11.6 & 96 & 83 & 46.6 & 98 & 84 & 3.1 \\
\hline
\end{tabular}

Table 2: SMT benchmarks

\begin{tabular}{|c|c|c|c|c|c|c|c|c|c|c|c|}
\hline & dplln & zchaff & & dplln & zchaff & & Cc & verit & & $C C$ & verit \\
\hline$H_{7}$ & 28.0 & 0.2 & $d e b$ & 111.5 & 0.8 & $F(1$ & 0.5 & 0.1 & $D_{5}$ & 2.3 & 0.3 \\
\hline$H_{8}$ & 262.7 & 1.2 & $d e b_{800}$ & 47.9 & 0 & $F(2$ & 1.3 & 0.1 & $D_{8}$ & 24.9 & 1.1 \\
\hline$H_{9}$ & - & 1.6 & $d e b_{900}$ & 201.6 & 1.2 & $F(25,15,5)$ & 0.5 & 0.2 & $D_{10}$ & 118.7 & 2.2 \\
\hline$H_{10}$ & _ & 6.7 & $d e b_{1000}$ & 260.4 & 1.5 & $F(25,24,24)$ & 16.9 & 0.1 & $D_{15}$ & - & 45.7 \\
\hline
\end{tabular}

Table 3: Comparison with Ergo in Coq 
sizes of Z3 proof witnesses is $12 \mathrm{Mb}$, and the mean of the sizes of veriT proof witnesses is $7.7 \mathrm{Mb}$. Table 2 presents the number of benchmarks solved by Z3 and veriT, and among them, the number of proof witnesses successfully checked by Isabelle/HOL and Coq. The times are the mean of the times for the benchmarks on which Z3, veriT, Isabelle/HOL and Coq all succeeds, in seconds. Errors in Coq were due to timeouts, and in Isabelle/HOL to timeouts and failures.

It appears that Coq can check a large part of the proof witnesses given by veriT (98.6\%) whereas Isabelle/HOL can check $88.0 \%$ of the proof witnesses given by Z3. As a result, even if Z3 can solve more benchmarks than veriT, the number of benchmarks solved by veriT combined with Coq is greater than the number of benchmarks solved by Z3 combined with Isabelle/HOL. Moreover, our combination is faster than the combination of $\mathrm{Z} 3$ with Isabelle/HOL. These results can be explained in great part by the fact that veriT gives much smaller proof witnesses. For instance, for logic QF IDL, in average, Z3 proof witnesses are 7.9 times bigger than veriT proof witnesses in terms of storing. The quality of veriT proof witnesses strengthens the fact we use it, even if there exists currently more performing SMT solvers. We have been told that the limitation of proof witnesses for LIA should disappear soon.

The four curves on the right of Figure 1 present the number of benchmarks solved along the time by the solvers and their combinations. They clearly indicate that our approach compares well with respect to [6].

Tactics We compare our zchaff and verit tactics with the reflexive tactics dplln and cc from Stephane Lescuyer's SMT solver Ergo written in Coq. To do so, we use the same formulas that are presented in Section 11.2 of [10]:

- for SAT:

- the famous pigeon hole formulas which are unsatisfiable

- the de Bruijn formulas: $\operatorname{de}_{n}=\forall x_{0}, \ldots, x_{2 n},\left(x_{2 n} \leftrightarrow x_{0}\right) \vee \bigvee_{i=0}^{2 n-1}\left(x_{i} \leftrightarrow x_{i+1}\right)$

- for EUF:

- the formulas $F P(n, m, k)=\forall f x, f^{n}(x)=x \rightarrow f^{m}(x)=x \rightarrow f^{k}(x)=x$ which are true for any $n, m, k$ such that $k$ is a multiple of $\operatorname{gcd}(n, m)$

- the formulas $D_{n}=$ $\forall f,\left(\bigwedge_{i=0}^{n-1}\left(x_{i}=y_{i} \wedge y_{i}=f\left(x_{i+1}\right)\right) \vee\left(x_{i}=z_{i} \wedge z_{i}=f\left(x_{i+1}\right)\right)\right) \rightarrow x_{0}=f^{n}\left(x_{n}\right)$

Results are presented in Table 3. Times are in seconds. We see that our zchaff and verit tactics here clearly outperform dplln and cc. This is not surprising since ZChaff and veriT have more efficient algorithms than Ergo. Note it may be difficult to change Ergo's algorithm since it would involve redoing many correctness proofs; the certificate approach is more flexible here. If we store proof witnesses, zchaff and verit get faster at rather small storage cost: in our examples, the largest proof witness is $41 \mathrm{Mb}$ large for $D_{15}$.

Regarding other existing Coq tactics, zchaff is far faster than tauto, and verit is similar to congruence. However, these latter ones do not solve the 
same goals, since verit can solve goals including congruence and propositional reasoning, and congruence can deal with inductive data-types.

\section{Conclusion and future works}

Compared to what the authors call "proof reconstruction" in [6], what we have presented here is much closer to program verification. We have developed inside Coq a program that checks traces generated by SAT and SMT solvers. A particular care has been given to the efficiency of the data representation for clauses and atoms. Even with the limited computing power available inside Coq, the checker is rather efficient: it is able to check in reasonable time huge proof witnesses coming from challenging benchmarks and it compares well with state of the art implementations in Isabelle/HOL.

From the methodology point of view, what we have done is very close to [13]. In this work, the authors have developed a checker for SMT proofs using LFSC. The main difference is that they delegate the verification of the SAT part to an external checker (written in $\mathrm{C}++$ ). Here, we do everything within the logic.

We also took a special care in being generic: for example the same checker is used for ZChaff and veriT. This relies on the generic format we use after translating proof witnesses into certificates. So we expect the checker to be easily extensible. For the moment, veriT is the only SMT solver that is connected with Coq. We hope that our format of certificate could also be used successfully to connect to other proof producing solvers. Our next step is to integrate Z3.

The checker has also been proved correct. Using the technique of proof by reflection, it made it possible to derive a safe and automatic proof procedure within Coq. Formulas usually proved in Coq are rather small so a far less efficient checker could have been sufficient. Still, we believe that our work opens interesting new perspectives of using brute force methods for doing proof with Coq automatically. For example, one could encode the small problem she/he has to prove as a huge Boolean formula that our SAT tactic can solve instantaneously.

Surprisingly the difficult part of this work was more the actual design of the certificate and obtaining a good computational behaviour for the checker than performing the correctness proofs. This is largely due to the fact that we are not proving the full functional correctness of the checker. We are just proving that if the checker replies true, the theorem is valid. This makes a big difference for the proof effort. This reduces drastically the size of the invariants we had to prove and clearly makes the proof of such a large piece of code tractable in Coq.

For future works, our priority is clearly to increase the expressiveness of the formulas we can deal with. In particular, if we want our tool to be widely used by the Coq community, being able to deal with quantified formulas and userdefined functions is a must-have. For quantifiers, it has not been done yet mostly because the current version of veriT does not produce proof witnesses. Though, this should be available in the next version of the system. For definitions, more work has to be done since the type system of Coq is more powerful than the 
one proposed by the SMT-LIB standard. Other extensions we envision concern non-linear arithmetic, arrays and bit vectors.

Acknowledgments Pascal Fontaine's responsiveness was crucial for this work. We wish to thank Sascha Böhme and Tjark Weber for the details and source code they gave us to reproduce the Isabelle experiments. We finally thank Christine Paulin, Guillaume Melquiond and Sylvain Conchon for their help concerning the comparison with dplln and cc in Coq. Anonymous referees provided helful constructive remarks.

\section{References}

1. Source code of the development, http://www.lix.polytechnique.fr/ keller/ Recherche/smtcoq.html

2. SMT-LIB, http://www.smtlib.org

3. Armand, M., Grégoire, B., Spiwack, A., Théry, L.: Extending Coq with Imperative Features and Its Application to SAT Verification. In: Kaufmann and Paulson [9], pp. $83-98$

4. Barendregt, H., Barendsen, E.: Autarkic Computations in Formal Proofs. J. Autom. Reasoning 28(3), 321-336 (2002)

5. Besson, F.: Fast Reflexive Arithmetic Tactics the Linear Case and Beyond. In: TYPES. LNCS, vol. 4502, pp. 48-62. Springer (2006)

6. Böhme, S., Weber, T.: Fast LCF-Style Proof Reconstruction for Z3. In: Kaufmann and Paulson [9], pp. 179-194

7. Dénès, M.: Coq with native compilation, https://github.com/maximedenes/ native-coq

8. Fontaine, P., Marion, J.Y., Merz, S., Nieto, L., Tiu, A.: Expressiveness + Automation + Soundness: Towards Combining SMT Solvers and Interactive Proof Assistants. In: TACAS. LNCS, vol. 3920, pp. 167-181. Springer (2006)

9. Kaufmann, M., Paulson, L.C. (eds.): Interactive Theorem Proving, First International Conference, ITP 2010, Edinburgh, UK, July 11-14, 2010. Proceedings, Lecture Notes in Computer Science, vol. 6172. Springer (2010)

10. Lescuyer, S., Conchon, S.: Improving Coq Propositional Reasoning Using a Lazy CNF Conversion Scheme. In: FroCos. LNCS, vol. 5749, pp. 287-303. Springer (2009)

11. McLaughlin, S., Barrett, C., Ge, Y.: Cooperating Theorem Provers: A Case Study Combining HOL-Light and CVC Lite. ENTCS 144(2), 43-51 (2006)

12. Nieuwenhuis, R., Oliveras, A., Tinelli, C.: Solving SAT and SAT Modulo Theories: From an abstract Davis-Putnam-Logemann-Loveland procedure to DPLL(). J. ACM 53(6), 937-977 (2006)

13. Oe, D., Stump, A.: Extended Abstract: Combining a Logical Framework with an RUP Checker for SMT Proofs. In: Lahiri, S., Seshia, S. (eds.) Proceedings of the 9th International Workshop on Satisfiability Modulo Theories (Snowbird, USA) (2011)

14. Tseitin, G.S.: On the complexity of proofs in propositional logics. Automation of Reasoning: Classical Papers in Computational Logic (1967-1970) 2 (1983)

15. Weber, T.: SAT-based Finite Model Generation for Higher-Order Logic. Ph.D. thesis, Institut für Informatik, Technische Universität München, Germany (Apr 2008), http://www.cl.cam.ac.uk/ tw333/publications/weber08satbased.html 